\title{
Telemedicine: a solution to disparities in human immunodeficiency virus prevention and pre-exposure prophylaxis uptake, and a framework to scalability and equity
}

\author{
Keith Yiu Kei Wong ${ }^{1}$, Chrysovalantis Stafylis $^{2}$, Jeffrey D. Klausner ${ }^{2}$ \\ ${ }^{1}$ David Geffen School of Medicine at UCLA, Los Angeles, CA, USA; ${ }^{2}$ Division of Infectious Diseases, Department of Medicine, University of \\ California Los Angeles, CA, USA \\ Contributions: (I) Conception and design: KYK Wong, JD Klausner; (II) Administrative support: KYK Wong, C Stafylis; (III) Provision of study \\ material or patients: C Stafylis, JD Klausner; (IV) Collection and assembly of data: KYK Wong; (V) Data analysis and interpretation: KYK Wong, JD \\ Klausner; (VI) Manuscript writing: All authors; (VII) Final approval of manuscript: All authors. \\ Correspondence to: Keith Yiu Kei Wong. David Geffen School of Medicine at UCLA, Los Angeles, CA, USA. Email: ywong@mednet.ucla.edu.
}

\begin{abstract}
As the group with the highest risk and the lowest pre-exposure prophylaxis (PrEP) utilization, black and Hispanic young men who have sex with men (MSM) face the brunt of the current human immunodeficiency virus (HIV) epidemic within the United States. In this narrative review, we summarized recent interventions that utilize technology (telemedicine) to improve HIV PrEP uptake. We examined the general model of those telemedicine programs and evaluated their success and limitations in addressing PrEP accessibility for at-risk individuals. We also identified the current bottlenecks in the scalability of telemedicine programs, offered suggestions to overcome these barriers, and advocated for a centralized source of funding to increase the accessibility of telemedicine programs for PrEP.
\end{abstract}

Keywords: Telemedicine; pre-exposure prophylaxis (PrEP); human immunodeficiency virus (HIV); access; disparity

Received: 19 July 2019; Accepted: 05 December 2019; Published: 05 April 2020.

doi: 10.21037/mhealth.2019.12.06

View this article at: http://dx.doi.org/10.21037/mhealth.2019.12.06

\section{Biomedical prevention for human immunodeficiency virus (HIV) in the United States}

Since the Food and Drug Administration's (FDA) approval of Truvada (emtricitabine/tenofovir disoproxil fumarate) for the use of pre-exposure prophylaxis (PrEP) in 2012, the annual rate of HIV diagnosis in the United States has been decreasing, from 13.2 per 100,000 population per year in 2012 to 11.8 in 2017 (1). However, the annual number of diagnoses remains high with 38,739 new cases in 2017 (1). Socioeconomic disparities in HIV continue to persist, with the highest infection burden being placed upon young men who have sex with men (MSM) of color (2). This pattern of socioeconomic disparities is also reflected in PrEP uptake, which remains low amongst those who are most at risk for HIV. PrEP users are more likely to be white $(3,4)$, between the age of 35-44 years old $(5,6)$ and have private insurance rather than Medicaid or to be uninsured (6). Although PrEP use among MSM in the United States has increased from $5.7 \%$ in 2014 to $35 \%$ in 2017 , uptake in the black and Hispanic/Latino MSM communities lags behind (7). In other words, PrEP is not reaching those who need it the most—our young MSM of color.

Many structural factors contribute to the disparities in PrEP uptake in the United States. Documented barriers to PrEP use and adherence include stigma, financial cost, healthcare system inaccessibility, fear for side effects, competing stressors, and low HIV risk perception (2,8-11). For individuals who are aware of their own HIV risk and the efficacy of PrEP, fear of homophobia from healthcare providers and potential outness to friends and family remains one of the main roadblocks that prevent patients from seeking care. Following along the PrEP care continuum, amongst those with high acceptability 
of PrEP, logistical concerns regarding the feasibility of obtaining PrEP due to financial cost and inability to access the healthcare system serve as another hurdle to PrEP utilization (12). Oftentimes, PrEP-providing clinics are unevenly distributed geographically, with less service available in areas with higher levels of poverty and higher proportions of black and Hispanics individuals (13). Unlike the current effort to treat HIV, there is an absence of dedicated federal funds for PrEP care and an integrated program for PrEP delivery (12). Patients who are uninsured or underinsured rely mainly on Medicaid expansion, pharmaceutical patient assistance programs, and a few statelevel PrEP assistance programs (PrEP-AP) to obtain the medication for PrEP (14), thus furthering the disparities in PrEP accessibility.

With the growth of wireless services and video conferencing technology, there is an expansion of various telemedicine programs to increase healthcare accessibility. As defined by Health Resource and Service Administration, telehealth is "the use of electronic information and telecommunications technologies to support long-distance clinical health care, patient and professional health-related education, public health, and health administration" (15). In this narrative review, we aim to examine recent approaches to telehealth and PrEP delivery in the United States, summarize these technology-based innovations, and evaluates their strengths and limitations. The goal is to provide an understanding of current models of telemedicine for PrEP, identify bottlenecks in telehealth accessibility and potential solutions to circumvent these barriers, and provide insights into the replicability and scalability of these interventions to better inform future HIV prevention strategies and their implementations.

\section{Methodology}

We first began by searching for PubMed studies and meeting abstracts from the 2019 National HIV Prevention Conference (NHPC) for the following key phrases: telemedicine, telehealth, mHealth, PrEP, and HIV. We only included previous and ongoing studies published in English and conducted in the United States. We excluded any study that does not provide PrEP medication prescription and delivery; for example, many telemedicine programs use technology to increase screening, awareness, education, and adherence for PrEP but did not describe a process for patients to obtain the medication. After initial screening and eligibility assessment, two published studies and two abstracts are included in this narrative review (Figure 1).

\section{Current strategies in telemedicine for PrEP}

The four studies in this narrative review include one pilot study (16) and three retrospective evaluations (17-19) on telemedicine interventions for PrEP implemented in private and public sectors across urban and rural areas. Telehealth interventions by academic centers and public health departments include the Iowa TelePrEP program (17) and the UCLA-led PrEPTech (16) program in the San Francisco Bay Area. Other for-profit, commercially available entities such as Nurx (18) and PlushCare (19) offer telemedicine for PrEP in the private sector.

These four reported telehealth programs use technology to reduce stigma, address privacy concerns, and improve access to resources in three key components of PrEP carepatient-to-provider consultations for PrEP initiation (20), PrEP-related laboratory tests, as well as PrEP prescription and delivery.

Generally, these programs operate on either a website or a mobile application that is accessible on any smartphone, tablet, or computer. Initial patient-to-provider consultations are done remotely through either a Health Insurance Portability and Accountability Act (HIPAA) compliant video messaging platform $(16,19)$, telephone call $(17)$, or a HIPAAcompliant text messaging platform (18). Additional followup and communication are also completed in a similar manner (16-19,21-29). While the modality of the visits varies among each program, what is crucial is the ability for patients to interact with a PrEP-friendly provider privately and conveniently at a time and setting of their choice.

Following the initial consultation, laboratory testing for HIV, common sexually transmitted infections (STIs) such as Neisseria gonorrboeae, chlamydia trachomatis, syphilis, and hepatitis B, as well as serum creatinine level are then performed before the prescription of PrEP. For some programs, patients are referred to a local outpatient clinic, laboratory, or public health facility for bloodwork and specimen collection (16). Other programs have explored the option to utilize at-home self-test kits (17-19). These self-test kits are mail-delivered to patients and require patients to either drop off the specimens in-person at a local laboratory $(17)$ or to mail them back for testing $(18,19)$ Once the laboratory tests are reviewed and the prescription has been approved, PrEP is either home-delivered (16-18) or made available to be picked up at a local pharmacy $(16,18,19)$. 


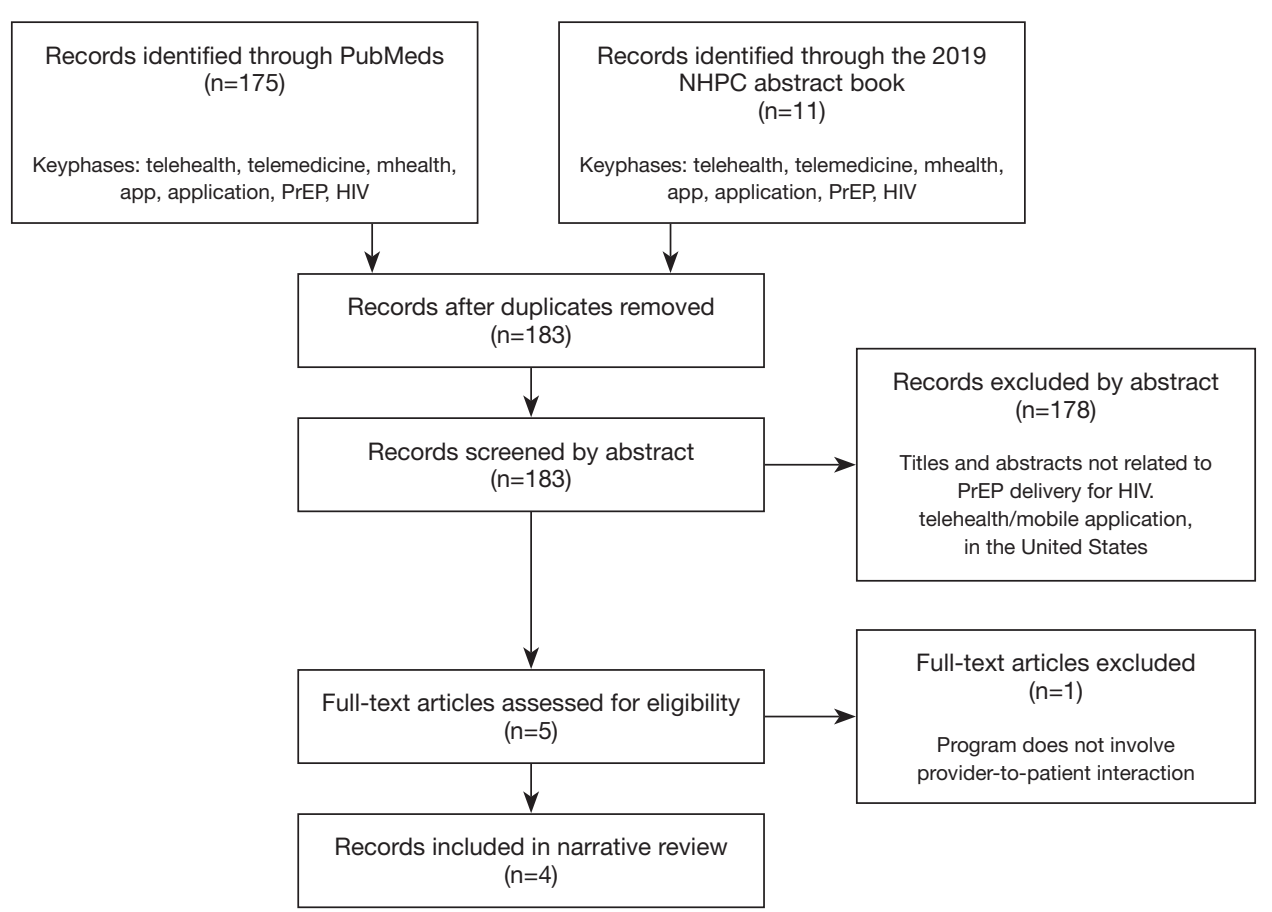

Figure 1 Flow diagram of study selection. NHPC, National HIV Prevention Conference; PrEP, pre-exposure prophylaxis; HIV, human immunodeficiency virus.

Although the general model of telemedicine for PrEP among those programs, from initial enrollment to medication delivery, is very similar (Figure 2), the financial cost associated with PrEP care can vary greatly. For the grant-supported PrEPTech study, the process was completely free, and care was offered at no cost to the participants (17). For other programs, PrEP is usually covered through a wide variety of sources-including private insurance, Medicaid, state-level or city-level PrEP-AP, the Gilead Advancing Access ${ }^{\circledR}$ Co-pay Program, and the Gilead Truvada for PrEP $^{\circledR}$ Medication Assistance Program. Almost all telehealth programs will offer a degree of PrEP navigation to connect patients to resources to pay for $\operatorname{PrEP}(16,18,19)$. The coverage for laboratory tests and appointment costs also varies depending on the patient's insurance status. Unlike the cost for medication, there is not a national assistance program that covers the fees for laboratory tests and physician appointment visits. Those who do not qualify for Medicaid will either have to apply for local-level PrEP-AP, which is currently found in ten states including California, Colorado, Washington DC, Florida, Illinois, Massachusetts, New York State, Ohio, Virginia, and Washington State (14), or pay out-of-pocket. For commercially available PrEP telemedicine programs, costs vary depending on the insurance status, starting from $\$ 100-130$ for the initial consultation visit for uninsured participants to only a small copay for insured participants $(22,23)$.

\section{Successes and limitations}

The initial findings of these telemedicine interventions under review show feasibility and promise in reaching individuals who are at risk of HIV in the United States (Table 1). Telemedicine is embraced by a racially diverse population-around $50 \%$ of the participants in the Nurx PrEP programs (18) and $48 \%$ of the PrEPTech study are black or Hispanic (17). In terms of age, participants in telemedicine programs have a mean age of 22, 31, 35 years old $(16,17,19)$, respectively, encompassing the young adult group (24-34 years old) who are most at-risk for HIV (2). While participants in the Iowa TelePrEP program are mostly white, nearly $83 \%$ of their participants live in small urban and rural areas, suggesting that telemedicine can be a useful tool in increasing PrEP availability for populations living in remote settings where resources are geographically-dispersed and difficult to access (16). Finally, these telemedicine programs for PrEP also demonstrate potentials when considering individual steps of the PrEP 


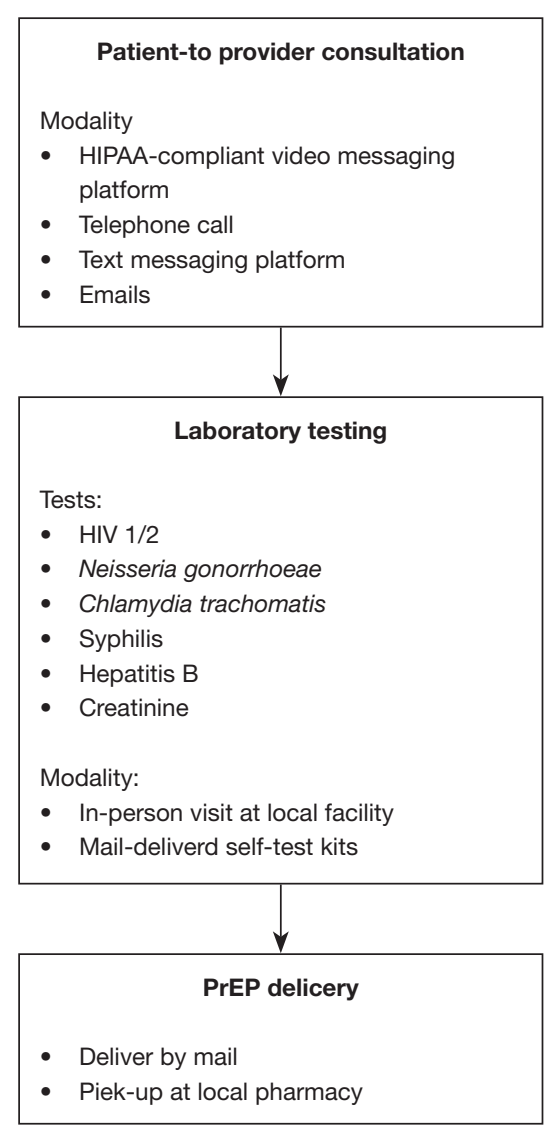

Figure 2 General schematic of telemedicine for PrEP programs. HIPAA, Health Insurance Portability and Accountability Act; PrEP, pre-exposure prophylaxis; HIV, human immunodeficiency virus.

care continuum. The percentage of PrEP initiation after the first telehealth appointment ranges from $84-94 \%(16,17)$ and 6-month retention remains relatively high, in the range of $76.2-98.7 \%(17,19)$.

The early success of these telemedicine programs for PrEP could be attributed to the ability of technology to address the barriers of geographic distance and social stigma faced by those who would otherwise have limited access to care. The use of telemedicine for PrEP is generally viewed by users as easy, fast, and convenient (17). The intrinsic familiarity to technology amongst a younger age group could also contribute to this perception. In addition, it is important to acknowledge that deliberate effort is sometimes needed to ensure these interventions can reach at-risk populations with different needs and characteristics. For the TelePrEP program in Iowa, attention was given to ensure the technology could perform in a low-bandwidth

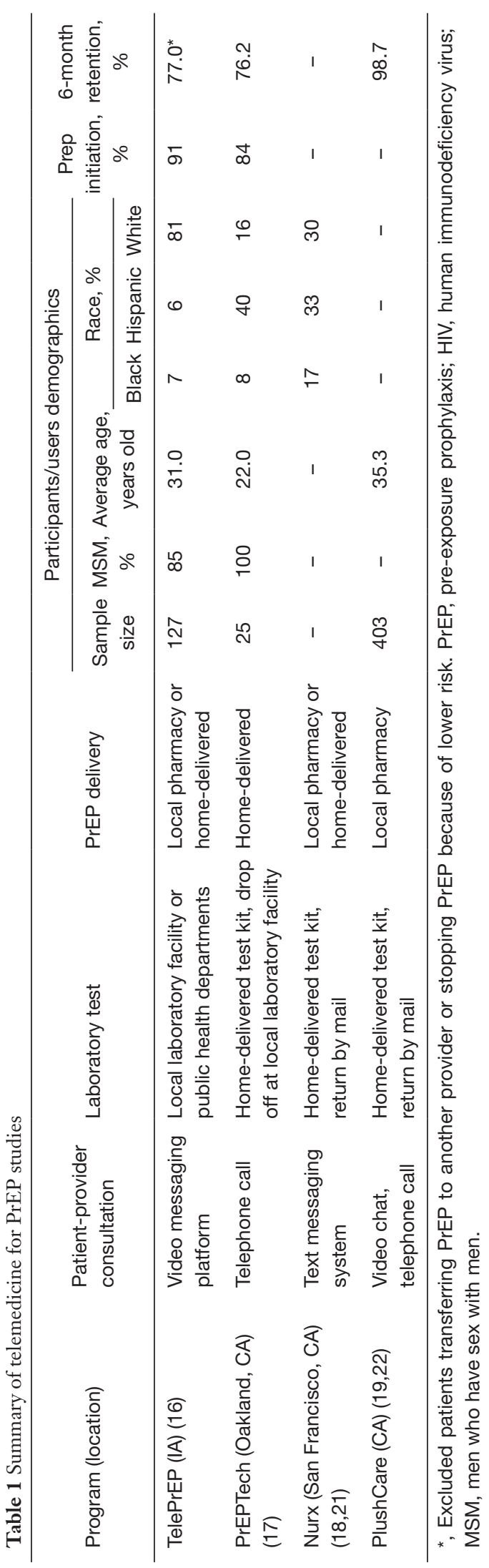


setting in rural communities (16); in a similar vein, for Nurx and the PrEPTech study, outreach efforts and advertising campaigns were intentionally designed with input from community focus groups to attract a racially diverse population $(17,18)$.

However, these optimistic findings are not without limitations. The telemedicine programs included have relatively small sample sizes, and the study populations vary in terms of race and urbanicity, thus potentially overestimating the impact and generalizability of these interventions. In addition, the absence of a control group limits the internal validity of these programs, as one could not compare these outcomes to those who do not have access to telemedicine services. Other socioeconomic factors such as income should also be taken into consideration when evaluating the accessibility of commercial programs such as Nurx (18) and PlushCare (19). Furthermore, these studies have limited data on adherence beyond 6-month after enrollment, thus limiting our ability to assess the long-term sustainability and impact of these interventions.

When assessing these programs as a whole, funding and cost remain one of the most prohibiting elements in the accessibility and scalability of telemedicine for PrEP (20). Partnerships between academic centers, community organizations, and state-level public health departments serve as the model for PrEP telehealth programs in the public sector. As an example, the Iowa TelePrEP program relies on the Iowa Department of Public Health for initial funding and utilizes on Medicaid expansion to cover PrEPassociated lab courses for uninsured individuals (16). Since PrEP coverage and patient assistance programs vary among states and there is not a centralized, federal fund dedicated to HIV prevention and PrEP availability, the process of creating a large-scale telehealth PrEP program remains difficult and depends heavily on state-level initiatives and local collaboration. Although for-profit entities such as Nurx and PlushCare have a much wider geographic network across the United States, with the former being available in 25 states as well as Washington DC (23) and the latter in all 50 states (24), their laboratory and appointment fee can be prohibitive for public utilization, especially among those who are uninsured.

\section{Suggestions for future models and research}

With laboratory testing and appointment cost being a barrier to accessing PrEP via telemedicine, a way to scale- up the current telehealth PrEP program for high-risk populations is for governments and other public entities to fill this financial gap and leverage the technology and infrastructure already built by these commercial telemedicine organizations. Recently, the state of California has attempted to achieve this goal.

In July 2019, the California Department of Public Health Office of AIDS initiated a collaboration with PlushCare to provide PrEP via telemedicine for patients enrolled in the state-operated PrEP-AP and reduce the geographic barriers to PrEP-AP clinician networks $(25,26)$. Under this model, patients will need to make a one-time in-person visit to a PrEP-AP enrollment site (27). Once eligible and enrolled, the patient will then attend either a video or phone consultation with a PrEP-AP provider using the PlushCare website or mobile app, complete the necessary laboratory tests in a local laboratory, and receive their medications in an in-network pharmacy (27). For uninsured individuals, their medication will be provided for free by the drug manufacturer and additional PrEP-related medical out-ofpocket costs will be covered by the PrEP-AP program (27). For insured individuals, the state program will pay for any additional cost for the medication and PrEP-related services after insurance coverage and the drug manufacturer's medication assistance program (27).

Although California PrEP-AP's telemedicine program is in its nascent stage and the structure of this partnership will require further evaluation for its long-term effectiveness, it serves as a possible model on how one can harness the current technological infrastructure to increase PrEP accessibility. However, what remains central to this discussion is the lack of dedicated federal investment in PrEP accessibility. In 2018, the Health Resources and Services Administration's (HRSA) Ryan White HIV/ AIDS Program provides 2.31 billion dollars of funding to treat and support individuals infected with HIV, especially through the AIDS Drug Assistance Program (ADAP) $(28,30)$. The program was founded under the Ryan White Comprehensive AIDS Resources Emergency Act (Ryan White CARE Act) of 1990, which was written "to improve the quality and availability of HIV care and treatment for low-income, uninsured, and underserved people living with HIV" (28). Despite undergoing several amendments and reauthorization, the act did not allow funds to be allocated for the prevention of HIV. Currently, only $3 \%$ of the U.S. federal funding for HIV/AIDS is dedicated to domestic HIV prevention (29) and there is not a PrEP-equivalent for the ADAP at the federal level $(12,30)$. Thus, those who are 
in need of PrEP rely on a patchwork of assistance programs that are tremendously difficult to navigate, which, when compounded by geographic distance and social stigma, further dampens PrEP uptake.

In 2015, PrEP accessibility was announced as one of the cornerstones of the CDC's Division of HIV/ AIDS Prevention's (DHAP) Strategic Plan to prevent new infections in 2020 (31). If that is indeed the goal, then the federal government and legislature will need to act to follow through with this vision. On March 8, 2019, New Jersey congresswoman Bonnie Watson Coleman introduced the PrEP-AP act that would establish a grant program through the federal Department of Health and Human Services to provide financial coverage for the costs of PrEP medication, laboratory fees, as well as PrEP outreach and counseling (32). This legislation, which currently has 38 cosponsors in the U.S. House of Representative, would allow those who are deterred by the cost associated with laboratory tests and appointments in telemedicine for PrEP to receive care (32). It offers a clear solution to the financial bottleneck within the current paradigm of telemedicine for PrEP and has the potential to transform the landscape of HIV prevention. With the initial success and promise of telehealth to bridge the gap of PrEP care in young MSM and individuals of color, there is a tremendous opportunity to reduce the disparities in PrEP utilization and end the spread of HIV.

\section{Limitation to this narrative review}

Our narrative review summarized the current literature on telemedicine interventions for PrEP delivery in the United States, their initial findings, and potential barriers to accessibility and scalability. However, there are several limitations to our approach. First, we did not conduct a systematic review, thus potentially excluding studies that could have affected our results. Second, since we only included studies conducted in the United States, the scope and applicability of our findings could be limited. Third, since the field of telehealth interventions for PrEP is still relatively nascent, our reliance on published data may offer a limited picture of the current strategies, as there may be ongoing studies and community programs with unpublished data that could affect our assessments. Finally, while our review attempted to identify the current financial barriers that hinder access to these telemedicine programs, we were not able to find studies on the cost-effectiveness of these interventions. Additional research on this topic is needed to better implement future telemedicine interventions for PrEP.

\section{Conclusions}

The current HIV epidemic disproportionally affects young black and Hispanic MSM; they are the most at-risk and yet have the lowest level of PrEP utilization. Novel telemedicine interventions in both the public and private sectors offer a promising solution to the geographic and societal barriers to PrEP uptake, especially for those who need it the most. Already established programs can serve as a model to scale-up telemedicine for PrEP, but advocacy for a centralized funding source such as the PrEP-AP Act is essential for overcoming the financial barriers and increasing the overall accessibility to PrEP care. Since these telehealth programs for PrEP are still relatively recent, there is a need for ongoing research to identify best practices for appointment modality, laboratory testing, and medication delivery, evaluate overall cost-effectiveness, as well as understand the long-term outcomes for PrEP retention and adherence.

\section{Key points on current telemedicine for PrEP interventions}

(I) The current telemedicine for PrEP programs utilizes a combination of communication technologies (telephone, mobile applications, websites, video conference calls) with laboratory testing in brickand-mortar sites or at-home STI/HIV test kits to overcome barriers to PrEP uptake.

(II) Telemedicine is a feasible tool for reaching young people and black and Hispanic MSM.

(III) Early research of telemedicine for PrEP interventions shows an encouraging level of PrEP initiation and 6-month retention among users.

(IV) The current bottleneck for telemedicine is the financial cost associated with laboratory tests and physician appointments. Centralized federal or state funding could cover those fees for the uninsured and underinsured.

(V) Future studies on best practices, cost-effectiveness, PrEP retention, and medication adherence are needed to understand the long-term benefits of telemedicine for PrEP. 


\section{Acknowledgments}

Funding: None.

\section{Footnote}

Conflicts of Interest: The authors have no conflicts of interest to declare.

Ethical Statement: The authors are accountable for all aspects of the work in ensuring that questions related to the accuracy or integrity of any part of the work are appropriately investigated and resolved.

Open Access Statement: This is an Open Access article distributed in accordance with the Creative Commons Attribution-NonCommercial-NoDerivs 4.0 International License (CC BY-NC-ND 4.0), which permits the noncommercial replication and distribution of the article with the strict proviso that no changes or edits are made and the original work is properly cited (including links to both the formal publication through the relevant DOI and the license). See: https://creativecommons.org/licenses/by-ncnd/4.0/.

\section{References}

1. Centers for Disease Control and Prevention. HIV surveillance report, 2017; vol. 29. Available online: http:// www.cdc.gov/hiv/library/reports/hiv-surveillance.html

2. Stahlman S, Lyons C, Sullivan PS, et al. HIV incidence among gay men and other men who have sex with men in 2020: where is the epidemic heading? Sex Health 2017;14:5-17.

3. Huang YA, Zhu W, Smith DK, et al. HIV Preexposure Prophylaxis, by Race and Ethnicity - United States, 20142016. MMWR Morb Mortal Wkly Rep 2018;67:1147-50.

4. Pulsipher CA, Montoya JA, Plant A, et al. Addressing PrEP disparities among young gay and bisexual men in California. Available online: https://aplahealth.org/wpcontent/uploads/2016/09/APLA_PrEP_FullReport_ WEB.pdf

5. Siegler AJ, Mouhanna F, Giler RM, et al. The prevalence of pre-exposure prophylaxis use and the pre-exposure prophylaxis-to-need ratio in the fourth quarter of 2017 , United States. Ann Epidemiol 2018;28:841-9.

6. Wu H, Mendoza MC, Huang YA, et al. Uptake of HIV preexposure prophylaxis among commercially insured
persons-United States, 2010-2014. Clin Infect Dis 2017;64:144-9.

7. Finlayson T, Cha S, Xia M, et al. Changes in HIV preexposure prophylaxis awareness and use among men who have sex with men - 20 urban areas, 2014 and 2017. MMWR Morb Mortal Wkly Rep 2019;68:597-603.

8. Wood S, Gross R, Shea JA, et al. Barriers and facilitators of PrEP adherence for young men and transgender women of color. AIDS Behav 2019;23:2719-29.

9. Ayala G, Makofane K, Santos GM, et al. Access to basic HIV-related services and PrEP acceptability among men who have sex with men worldwide: barriers, facilitators, and implications for combination prevention. J Sex Transm Dis 2013;2013:953123.

10. Bauermeister JA, Meanley S, Pingel E, et al. PrEP awareness and perceived barriers among single young men who have sex with men. Curr HIV Res 2013;11:520-7.

11. Jaiswal J, Griffin M, Singer SN, et al. Structural barriers to pre-exposure prophylaxis use among young sexual minority men: the P18 cohort study. Curr HIV Res 2018;16:237-49.

12. Mayer KH, Chan PA, Patel R, et al. Evolving models and ongoing challenges for HIV preexposure prophylaxis implementation in the United States. J Acquir Immune Defic Syndr 2018;77:119-27.

13. Siegler AJ, Bratcher A, Weiss KM, et al. Location location location: an exploration of disparities in access to publicly listed pre-exposure prophylaxis clinics in the United States. Ann Epidemiol 2018;28:858-64.

14. National Alliance of State and Territorial AIDS Directors (NASTAD). PrEP assistant programs. Private PrEP co-pay assistance programs for underinsured patients. Available online: https://www.nastad.org/prepcost-resources/prepassistance-programs

15. Health Resources and Services Administration. Telehealth programs. Available online: https://www.hrsa.gov/ruralhealth/telehealth/index.html

16. Refugio ON, Kimble MM, Silva CL, et al. Brief report: PrEPTECH: a telehealth-based initiation program for HIV pre-exposure prophylaxis in young men of color who have sex with men. A pilot study of feasibility. J Acquir Immune Defic Syndr 2019;80:40-5.

17. Hoth AB, Shafer C, Dillon DB, et al. Iowa TelePrEP: a public-health-partnered telehealth model for HIV preexposure prophylaxis (PrEP) delivery in a rural state. Sex Transm Dis 2019. [Epub ahead of print].

18. Horwitz J, Hawkins M, Koester K. Reducing barriers to PrEP access through innovative app and testing methods. Atlanta: 2019 National HIV Prevention Conference, 2019. 
19. Hung R, Bandy J, Wantuck J. PlushCare tele-PrEP, a nationwide app-based program's California success. Atlanta: 2019 National HIV Prevention Conference, 2019.

20. Touger R, Wood BR. A review of telehealth innovations for HIV pre-exposure prophylaxis (PrEP). Curr HIV/ AIDS Rep 2019;16:113-9.

21. Nurx. HIV Prevention FAQs. Available online: https:// www.nurx.com/faq/category/hiv-prevention-faqs

22. PlushCare. PrEP with PlushCare - frequently asked questions. Available online: https://www.plushcare.com/ blog/plushcare-prep-faq

23. Nurx. Where is Nurx available. Available online: https:// nurx.zendesk.com/hc/en-us/articles/115002900931Where-is-Nurx-available

24. Spera M. PlushCare goes nationwide, serving all 50 states via online doctors. Available online: https://www.plushcare. com/blog/plushcare-online-doctors-nationwide-serving50-states

25. McQuaid R. PlushCare Partners with the California Department of Public Health, Providing Free Access to PrEP Statewide. Available online: https://www.plushcare. com/blog/plushcare-partners-with-california-dph

26. Barraza A. Implementation plan for phase one (uninsured clients) of the preexposure prophylaxis assistance program (PrEP-AP). Office of AIDS, California Department of Public Health, California Health and Human Services Agency. Available online: https://www.cdph.ca.gov/ Programs/CID/DOA/CDPH\%20Document\%20

doi: $10.21037 /$ mhealth.2019.12.06

Cite this article as: Wong KYK, Stafylis C, Klausner JD. Telemedicine: a solution to disparities in human immunodeficiency virus prevention and pre-exposure prophylaxis uptake, and a framework to scalability and equity. mHealth 2020;6:21.
Library/2018-01\%20PrEP-AP\%20Implementation\%20 Plan.pdf

27. California Department of Public Health. Go-live announcement for prep-ap telemedicine provider. Available online: https://www.cdph.ca.gov/Programs/ CID/DOA/CDPH\%20Document\%20Library/ADAP _ MM_2019_12_Go_Live_PrEP_AP_Telemedicine_ Provider.pdf

28. Health Resources \& Services Administration. HRSA's Ryan White HIV/AIDS program: program overview. Program fact sheet. Available online: https://hab.hrsa.gov/ sites/default/files/hab/Publications/factsheets/programfactsheet-program-overview.pdf

29. Kaiser Family Foundation. U.S. Federal Funding for HIV/ AIDS: Trends Over Time. Available online: https://www. kff.org/hivaids/fact-sheet/u-s-federal-funding-for-hivaidstrends-over-time/

30. Health Resources \& Services Administration. Ryan White HIV/AIDS program funding. Available online: https:// hab.hrsa.gov/program-grants-management/ryan-whitehivaids-program-funding

31. Centers for Disease Control and Prevention. DHAP Strategic Plan. Available online: https://www.cdc.gov/hiv/ dhap/strategicplan/index.html

32. 116th Congress (2019-2020). H.R.1643 - PrEP assistance program act. Available online: https://www.congress.gov/ bill/116th-congress/house-bill/1643/ 